\title{
A comparative study on normal and obese mice indicates that the secretome of mesenchymal stromal cells is influenced by tissue environment and physiopathological conditions
}

Serife Ayaz-Guner ${ }^{1}$, Nicola Alessio², Mustafa B. Acar ${ }^{3,4}$, Domenico Aprile², Servet Özcan ${ }^{3,4}$, Giovanni Di Bernardo², Gianfranco Peluso ${ }^{5}$ and Umberto Galderisi ${ }^{2,3,6^{*}}$ (D)

\begin{abstract}
Background: The term mesenchymal stromal cells (MSCs) designates an assorted cell population comprised of stem cells, progenitor cells, fibroblasts, and stromal cells. MSCs contribute to the homeostatic maintenance of many organs through paracrine and long-distance signaling.

Tissue environment, in both physiological and pathological conditions, may affect the intercellular communication of MSCs.

Methods: We performed a secretome analysis of MSCs isolated from subcutaneous adipose tissue (SWAT) and visceral adipose tissue (VWAT), and from bone marrow (BM), of normal and obese mice.

Results: The MSCs isolated from tissues of healthy mice share a common core of released factors: components of cytoskeletal and extracellular structures; regulators of basic cellular functions, such as protein synthesis and degradation; modulators of endoplasmic reticulum stress; and counteracting oxidative stress. It can be hypothesized that MSC secretome beneficially affects target cells by the horizontal transfer of many released factors. Each type of MSC may exert specific signaling functions, which could be determined by looking at the many factors that are exclusively released from every MSC type.

The vWAT-MSCs release factors that play a role in detoxification activity in response to toxic substances and drugs. The sWAT-MSC secretome contains proteins involved in in chondrogenesis, osteogenesis, and angiogenesis. Analysis of BMMSC secretome revealed that these cells exert a signaling function by remodeling extracellular matrix structures, such as those containing glycosaminoglycans. Obesity status profoundly modified the secretome content of MSCs, impairing the above-described activity and promoting the release of inflammatory factors.
\end{abstract}

Conclusion: We demonstrated that the content of MSC secretomes depends on tissue microenvironment and that pathological condition may profoundly alter its composition.

Keywords: Obesity, Mesenchymal stromal cells, Secretome

\footnotetext{
* Correspondence: umberto.galderisi@unicampania.it

${ }^{2}$ Department of Experimental Medicine, Luigi Vanvitelli Campania University, Naples, Italy

${ }^{3}$ Genome and Stem Cell Center (GENKOK), Erciyes University, Kayseri, Turkey

Full list of author information is available at the end of the article
}

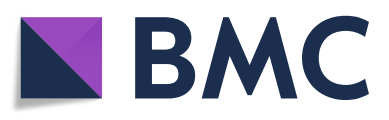

( The Author(s). 2020 Open Access This article is licensed under a Creative Commons Attribution 4.0 International License, which permits use, sharing, adaptation, distribution and reproduction in any medium or format, as long as you give appropriate credit to the original author(s) and the source, provide a link to the Creative Commons licence, and indicate if changes were made. The images or other third party material in this article are included in the article's Creative Commons licence, unless indicated otherwise in a credit line to the material. If material is not included in the article's Creative Commons licence and your intended use is not permitted by statutory regulation or exceeds the permitted use, you will need to obtain permission directly from the copyright holder. To view a copy of this licence, visit http://creativecommons.org/licenses/by/4.0/. The Creative Commons Public Domain Dedication waiver (http://creativecommons.org/publicdomain/zero/1.0/) applies to the data made available in this article, unless otherwise stated in a credit line to the data. 


\section{Background}

Mesenchymal stromal cells (MSCs) are an heterogeneous cell population comprised of stem cells, progenitor cells, fibroblasts, and stromal cells. MSCs reside in the stromal component of several tissues and organs, including bone marrow, cord blood, dental pulp, and adipose tissue. Stem cells present in MSCs can be differentiated into chondrocytes, osteocytes, adipocytes, and other mesodermal cell types. MSCs contribute to the homeostatic maintenance of many organs through paracrine and long-distance signaling [1]. For this reason, MSCs and their products are under scrutiny in numerous clinical trials, to treat several human diseases $[2,3]$.

MSCs within different tissues are exposed to peculiar microenvironments that impact their phenotypes and functions, with specific modulations of cell proliferation, differentiation, self-renewal, and survival. Many investigations have focused on the biology of bone marrowderived (BM) and white adipose tissue-derived (WAT) MSCs, since these tissue sources are the most used for isolating MSCs that are employed in cell therapy. Furthermore, BM and WAT resident MSCs play a key role in organismal physiopathology, given the wide distribution of these tissues within the body [1]. Some studies have shown that BM-MSCs and WAT-MSCs differ in their transcriptional profiles, surface antigen expressions, differentiation potentials, and biological functions, such as their effects on cancer cells [4-7]. Pathological conditions may alter the microenvironment surrounding MSCs a d impair their functions. Some findings have demonstrated that MSC dysfunctions are associated with several diseases, including diabetes, lupus, psoriasis, rheumatoid arthritis, and metabolic syndrome $[8,9]$.

Tissue environment, in both physiological and pathological conditions, may significantly affect the intercellular communication of MSCs, which occurs through cellcell contact, soluble factors (growth factors, hormones, cytokines, metabolites, etc.), and the release of extracellular vesicles (EVs). These vesicles range from 30 to $1000 \mathrm{~nm}$ and carry many bioactive molecules, surface receptors, and genetic information (DNA, diverse types of RNAs). EVs interact with target cells, which may be close to or distant from the originating cell. EV signaling can occur either through ligand-receptor interaction at the target cell's surface or through the fusion of vesicles with cell plasma membranes (endocytosis) [10].

MSC tissue homeostasis and regeneration activities occur mainly through the release of soluble factors and EVs that enter blood circulation and can reach target tissues throughout the body. This MSC property is also the basis of their therapeutic effectiveness in cell therapy treatments $[1,11]$.

In this context, the aim of our study was to evaluate how physiological and pathological changes in the MSC microenvironment affect secretome composition and hence MSC functions. We decided to perform an unbiased analysis of the entire proteome content of MSC secretome. Specifically, we collected and analyzed the secretomes of MSCs obtained from subcutaneous and visceral WAT, as well as from bone marrow, of normal and obese mice. Obesity leads to WAT dysfunction, promoting chronic inflammation and cardiovascular and metabolic pathologies. The body contains subcutaneous, visceral, and bone marrow fat depots, whose distribution and functions are altered in obese individuals [12, 13]. We chose obesity for the pathological condition, since this disease greatly affects the fat depots where MSCs reside.

\section{Material and methods \\ Animals}

Six C57BL/6 inbred male mice age 3 weeks were purchased from Charles River (Wilmington, MA, USA). As the study involved animals, it was approved by the Italian Ministry of Health (n. 317-2016PR), and mice were handled in compliance with the protocols approved by the Animal Care and Use Committee of University Campania Luigi Vanvitelli. After arrival, the mice were divided into two groups and were fed either a high-fat diet (HFD) (Research Diets, New Brunswick, NJ, USA) or a normal diet (ND) for 10 weeks. At the end of this treatment, the mice were euthanized, and tissue samples were harvested for the experiments laid out below.

The high-fat diet consisted of $60 \%$ fat from lard, $20 \%$ carbohydrates, and $20 \%$ protein (total $5.21 \mathrm{kcal} / \mathrm{g}$ ), whereas the normal diet consisted of $10 \%$ fat, $70 \%$ carbohydrates, and $20 \%$ protein (total $3.82 \mathrm{kcal} / \mathrm{g}$ ). Food intake and body weight were measured once a week until the end of the experiments.

\section{Glucose measurement}

At the end of high and normal fat treatments, blood glucose levels were determined in fasting mice by tail bleeding, using a Contour blood glucose meter (Ascensia Diabetes Care, Parsippany, NJ, USA) according to the manufacturer's instructions.

\section{MSC isolation and cultivation}

We harvested MSCs from the bone marrow of mice's femurs and tibias by inserting a 21-gauge needle into the shaft of the bone and flushing it with alpha-MEM. The cells from one mouse were plated onto two 100-mm dishes with alpha-MEM containing 15\% FBS. After $48 \mathrm{~h}$, we discarded the nonadherent cells and washed the adherent cells with PBS (phosphate-buffered saline) 1X. We then incubated the cells for 7 to 10 days in a proliferating medium in order to reach confluence (P0). Cells were grown until passage 3 for secretome harvest. 
We collected MSCs from $500 \mathrm{mg}$ of subcutaneous WAT (sWAT) surrounding the hips of the mice and from $1 \mathrm{~g}$ of visceral WAT (vWAT) obtained from the abdomen area. Tissues were digested for $1 \mathrm{~h}$ at $37^{\circ} \mathrm{C}$ in a DMEM solution containing collagenase type II $(1 \mathrm{mg} /$ $\mathrm{ml})$. Samples were filtered through cell strainers $(70 \mu \mathrm{m}$ mesh), centrifuged, and washed three times with PBS 1X. Cells were plated onto $100-\mathrm{mm}$ dishes with alphaMEM containing 15\% FBS. We then incubated the cells for 7 days in a proliferating medium in order to reach confluence (P0). Cells were grown until passage 3 for secretome harvest.

\section{Secretome harvest}

MSC cultures (80\% confluent) were washed extensively with PBS and then transferred to a chemically-defined, serum-free culture medium for overnight incubation. Then, the conditioned media containing MSC secretion were collected and used for liquid chromatography-mass spectrometry (LC-MS) analysis.

\section{Secretome preparation for LC-MS/MS analysis}

Five $\mathrm{mL}$ of secretomes were collected from culture dishes without disturbing the attached cells, at which point culture debris were removed by centrifugation at 10.000 g. Supernatants were used for StartaClean beads protein pooling. Dried beads were mixed with $1 x$ Laemmli gel loading buffer and run on a gradient gel 415\% SDS-PAGE (Criterion TGX Stain Free Precast Gels, BIO-RAD, USA). Electrophoresis was carried out at 100 V. After electrophoresis, gels were stained with Coomassie, and the gel lanes of interest were excised for in-gel digestion.

After digestion, the peptides were eluted from the gel matrix by immersion of the reaction tube in an ultrasonic bath for $5 \mathrm{~min}$, with sequential elution of $0.4 \%$ formic acid in $3 \% \mathrm{ACN}, 0.4 \%$ formic acid in $50 \% \mathrm{ACN}$, and $0.4 \%$ formic acid in $100 \%$ ACN. The supernatant containing the peptides was centrifuged, transferred to low binding tubes, and desalted with ZipTip C18 (Millipore, Merck). After that, the extracted peptides were dried and stored at $-80^{\circ} \mathrm{C}$ until the LC-MS/MS analysis.

\section{LC-MS/MS analysis}

Tandem mass spectrometric analysis was carried out using an $\mathrm{AB}$ SCIEX TripleTOF 5600+ instrument (AB SCIEX, Redwood City, CA, USA) coupled to an Eksigent expert nano-LC 400 system (AB SCIEX). MS and MS/ MS data were acquired using Analyst ${ }^{\circ}$ TF v.1.6 (AB SCIEX).

Mass spectrometry data was analyzed using ProteinPilot 4.5 Beta (AB SCIEX) for peptide identification.

\section{Gene ontology analysis}

The proteins expressed in the secretomes were analyzed using the PANTHER (Protein ANalysis THrough Evolutionary Relationships - http://www.pantherdb.org) software. In PANTHER, the protein classification was performed according to the ontology terms: cellular component, protein class, molecular function, biological processes, and pathway. For the PANTHER analysis, we used the statistics overrepresentation (default setting), comparing classifications of multiple clusters of lists to a reference list in order to statistically identify overrepresentation of PANTHER ontologies. The selected $p$-value was set at 0.05 . We followed the developers' instructions for running a PANTHER analysis [14].

\section{Pathway analysis}

Differentially-expressed proteins were imported into Reactome software for detailed pathway identification $[15,16]$. The Reactome Knowledgebase (https://reactome.org) provides molecular details of cellular processes as an ordered network of molecular transformations in a single consistent data model. We submitted LC/MS data as a single column of identifiers (UniProt IDs), and the software mapped them to pathways. Over-representation and pathway-topology analyses were conducted. Over-representation analysis is based on statistical hypergeometric distribution, and it evaluates whether certain specific Reactome pathways are enriched in the submitted data. This analysis produced a probability score, which was then corrected for false discovery rate (FDR) using the BenjamaniHochberg method. The FDR was set at $p<0.05$.

\section{Results}

We fed two groups of mice (three mice per group) with a high-fat diet (HFD) or a normal diet (ND) for 10 weeks. In the ND group, the average weight increased from $21.0 \pm 2.5 \mathrm{~g}$ to $26 \pm 2.3 \mathrm{~g}$, while in the HFD group, the weight started from $20.6 \pm 2.3 \mathrm{~g}$ rose to $44.2 \pm 4.5 \mathrm{~g}$. The HFD treatment induced hyperglycemia $(170 \pm 6.5$ $\mathrm{mg} / \mathrm{dL}$ in ND versus $280 \pm 15.5 \mathrm{mg} / \mathrm{dL}$ in HFD), determined by blood glucose measurement.

We then isolated and cultivated MSCs from BM, visceral WAT (vWAT), and subcutaneous WAT (sWAT) of both normal and obese mice to evaluate their in vitro properties. We verified by flow cytometry that MSCs expressed the surface antigens CD105, CD90, and CD73 and were able to differentiate into adipocytes, chondrocytes, and osteocytes (Additional file 1).

We grew MSCs in vitro until passage 3 and then collected secretomes for the analysis of their proteome content.

We had three biological replicates for each type of MSC culture (BM-MSC, sWAT-MSC, and vWAT-MSC 
secretomes); globally, we collected 18 secretome samples-9 from HFD-treated mice and 9 from ND-treated mice. We performed LC-MS/MS analyses on peptides from the tryptic digestion of secretome samples. Each sample had two technical replicates (Additional file 2).

We employed high-resolution MS in a search of the Protein Metrics database, wherein several hundred proteins were identified in all the experimental conditions (Additional file 2). We merged data from technical and biological replicates through a Venn diagram analysis, thereby obtaining a list of proteins expressed in the various experimental conditions (Table 1).

\section{Gene ontology (GO) analysis in samples from ND-treated mice}

GO implements an enrichment analysis of ontology terms in the proteomic profile of interest. An ontology term consists of a set of proteins with relations that operate between them. We matched our experimental data to reference ontology terms by using PANTHER's GO enrichment analysis, and we identified the ontology terms that were overrepresented in our datasets compared to a reference mouse protein set.

We focused our GO analysis on ontological terms belonging to the following GO domains (hierarchical biological clusters): cellular components, protein classes, molecular functions, biological processes, and pathways. For each experimental condition, we identified dozens of ontologies (Additional file 3). We then performed a Venn diagram analysis to combine the data of all experimental conditions in order to find both the specific and the common ontologies among the secretomes of BMMSCs, vWAT-MSCs, and sWAT-MSCs from NDtreated mice. The most representative ontologies are depicted in Tables 1 and 2.

Cellular component, protein class, and molecular function $\mathrm{GO}$ analyses demonstrated that proteins belonging to cytoskeleton and extracellular matrix (ECM) structures, those belonging to signaling networks, those belonging to the oxy-redox class, and those involved in protein anabolism/catabolism were overrepresented in the secretomes of MSCs from ND-treated mice (Table 2, Fig. 1). Of note, in the secretomes of BM- and sWATMSCs, we also identified proteins belonging to chaperone, growth factor, and cytokine families (Table 2, Fig. 1). Biological process and pathway GO analyses showed that proteins involved in actin nucleation, cell

Table 1 Number of proteins per secretome

\begin{tabular}{lll}
\hline & HFD & ND \\
\hline BM-MSCs & 444 & 487 \\
sWAT -MSCs & 510 & 573 \\
wWAT-MSCs & 381 & 257 \\
\hline
\end{tabular}

motility, protein synthesis, endoplasmic reticulum stress, oxidative stress, and metabolism were overrepresented in the secretomes of MSCs from ND-treated mice (Table 3, Fig. 1). In addition, the vWAT-MSCs secreted several proteins involved in responding to toxic substances and drugs, as well as proteins that play a role in the small molecule metabolic process. The secretomes of sWAT-MSCs and BM-MSCs contained proteins that regulate leukocyte and granulocyte chemotaxis, as well as negative regulators of cell death (Table 3). In BM-MSC secretome, many proteins were seen that are involved in metabolism (carbohydrate, pyruvate, and lipid metabolic processes) (Table 3). Of great interest, sWAT-MSCs released many factors that modulate proliferation and differentiation of several cell types involved in angiogenesis, chondrogenesis, and osteogenesis (Table 3).

\section{Gene ontology (GO) analysis in samples from HFD-treated mice}

We evaluated how obesity affected the GO ontologies of MSC-secreted proteins. Importantly, in samples from obese mice, we observed the absence of some GO terms found in normal mice and the presence of a few new ontologies (Tables 2 and 3). Specifically, in vWAT samples from HFD-treated mice, proteins involved in response to drugs and small molecule metabolism were absent. Additionally, factors involved in oxy-redox or transition metal ion binding activities were not found (Tables 2 and 3). In the sWAT-MSC secretome, several proteins associated with lipid metabolism and some growth factors were no longer present in samples from obese mice (Tables 2 and 3). Two new GO ontology groups were present in the sWAT-MSC secretome obtained from HFD-treated mice: response to interleukin-1 (IL-1) and cholecystokinin (CCK)B/gastrin receptors (CCKR) signaling map. IL-1 pathway is intensely activated during inflammation and may contribute to chronic inflammation, associated with obesity [17]. The gastrin cholecystokinin B receptors trigger signaling pathways, which influence the expression of genes that are involved in cell survival, angiogenesis, and invasion [18].

In the secretomes of BM-MSCs obtained from obese mice, several ontologies associated with metabolism and protein synthesis were absent. Of note, in these samples, we also observed GO terms associated with IL-1 pathway (Tables 2 and 3). BM-MSCs from obese mice released several proteins that modulate chondrogenesis and osteogenesis; these factors were absent in the secretome from normal mice.

\section{Reactome analysis in samples from ND-treated mice}

Experimental data analysis with GO gives a general view of the most significant ontology groups present in the datasets, but it cannot directly define the most important 
Table 2 .

\begin{tabular}{|c|c|c|c|c|}
\hline & Common GO among vWAT sWAT BM & GO vWAT specific & GO sWAT specific & GO BM specific \\
\hline \multicolumn{5}{|c|}{$\begin{array}{l}\text { COMMON AND SPECIFIC GENE ONTOLOGY (GO) ENTITIES IN ND SAMPLES } \\
\end{array}$} \\
\hline \multirow[t]{7}{*}{$\begin{array}{l}\text { GO CELLULAR } \\
\text { COMPONENT }\end{array}$} & Arp2/3 protein complex & & $\begin{array}{l}\text { Chaperonin containing } \\
\text { T-complex }\end{array}$ & $\begin{array}{l}\text { Chaperonin containing } \\
\text { T-complex }\end{array}$ \\
\hline & Actin filament & & & Lysosome \\
\hline & Extracellular space (ECM) & & & \\
\hline & Collagen containing ECM & & & \\
\hline & Cytosolic small ribosomal subunit & & & \\
\hline & Cytosolic large ribosomal subunit & & & \\
\hline & Proteasome core complex & & & \\
\hline \multirow[t]{11}{*}{ GO PROTEIN CLASS } & Non-motor actin binding protein & Peroxidase & Growth factor & Cytokine \\
\hline & Actin and actin related protein & Reductase & Metalloprotease & Metalloprotease \\
\hline & $\begin{array}{l}\text { Extracellular matrix structural } \\
\text { protein }\end{array}$ & & Nucleic acid binding protein & Serine protease \\
\hline & Oxidoreductase & & Transporter & Nucleic acid binding protein \\
\hline & Ribosomal protein & & & Transporter \\
\hline & Protease inhibitor & & & \\
\hline & Hsp90 family chaperone & & & \\
\hline & G protein coupled receptor & & & \\
\hline & Calmodulin-related & & & \\
\hline & Zinc finger transcription factor & & & \\
\hline & Immunoglobulins & & & \\
\hline \multirow{10}{*}{$\begin{array}{l}\text { GO MOLECULAR } \\
\text { FUNCTION }\end{array}$} & Extracellular matrix binding & Protein & Growth factor activity & Protein serine/threonine \\
\hline & Integrin binding & serine/threonine & Carboxypeptidase activity & \\
\hline & Structural constituent of ribosome & kinase activity & & \\
\hline & Protease binding & & & \\
\hline & Serine type endopeptidase & & & \\
\hline & Metalloendopeptidase & & & \\
\hline & Transition ion metal binding & & & \\
\hline & ATP binding & & & \\
\hline & G protein coupled receptor activity & & & \\
\hline & $\begin{array}{l}\text { Transmembrane transporter } \\
\text { activity }\end{array}$ & & & \\
\hline \multicolumn{5}{|c|}{ CHANGES IN HFD SAMPLES } \\
\hline $\begin{array}{l}\text { GO CELLULAR } \\
\text { COMPONENT }\end{array}$ & & & & Ribosomal protein ABSENT \\
\hline GO PROTEIN CLASS & & Peroxidase ABSENT & & \\
\hline \multirow[t]{2}{*}{$\begin{array}{l}\text { GO MOLECULAR } \\
\text { FUNCTION }\end{array}$} & & $\begin{array}{l}\text { Transition ion metal } \\
\text { binding ABSENT }\end{array}$ & $\begin{array}{l}\text { Transmembrane transporter } \\
\text { activity ABSENT }\end{array}$ & $\begin{array}{l}\text { Structural constituent of } \\
\text { ribosome ABSENT }\end{array}$ \\
\hline & & & Growth factor activity ABSENT & \\
\hline
\end{tabular}

proteins in the analyzed proteomes. However, this can be achieved with Reactome analysis. In this analysis, any event that modifies the state of a biological molecule is defined as a 'reaction'. Specifically, binding, activation, translocation, degradation, and all other biochemical events involving a catalyst are considered reactions [15, 16]. The assumption is that a given protein group found in the experimental data reflects a key functional importance for the phenotype(s) under analysis if all the proteins are part of the same Reactome pathway.

The secretome contents of vWAT-MSCs, sWATMSCs, and BM-MSCs from ND-treated mice were assigned to 27, 13, and 17 Reactome pathways, respectively (Table 4). Three pathways were in common among the secretomes: cross presentation of soluble antigens (endosomes); post-translational protein phosphorylation; 


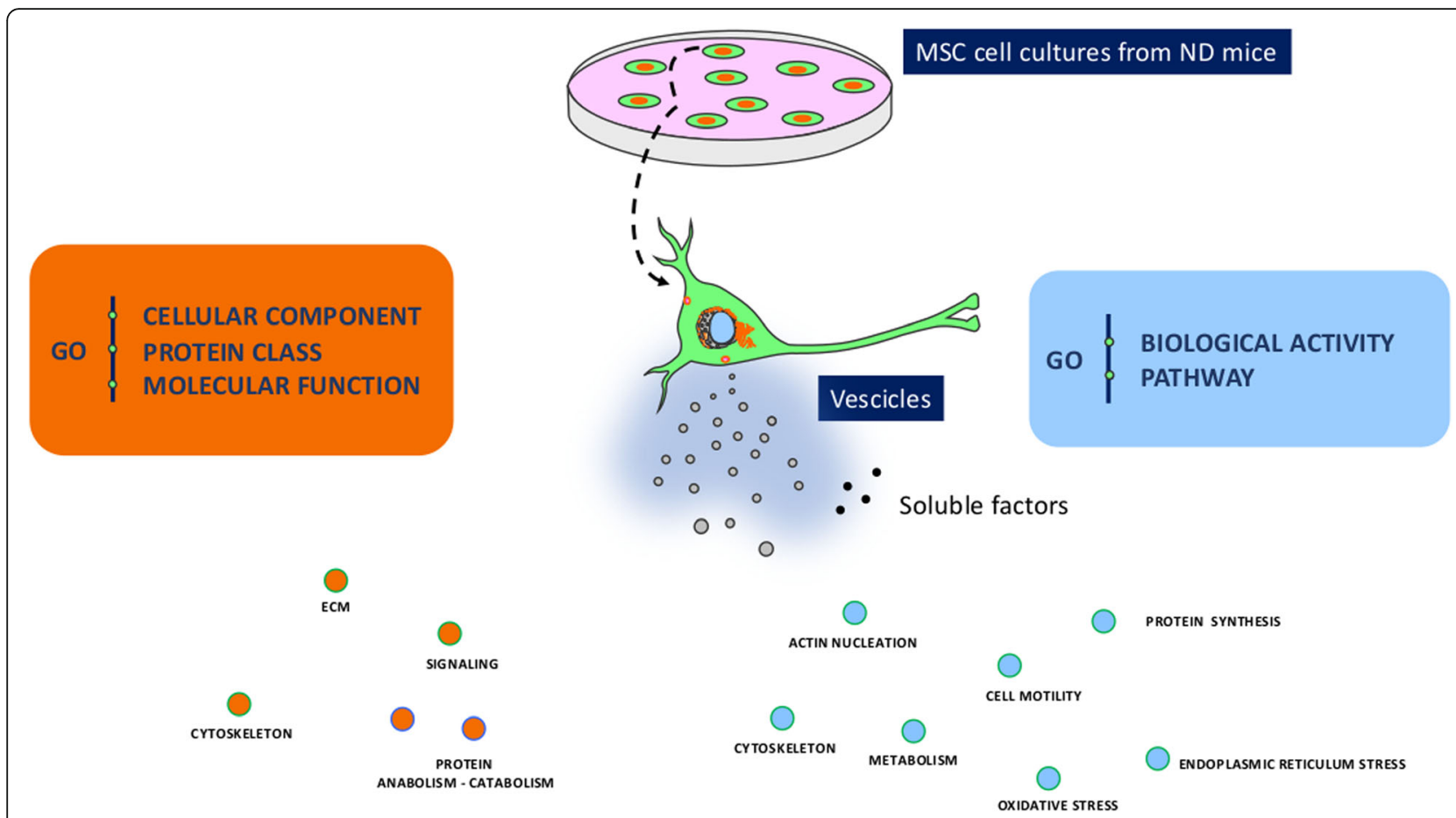

Fig. 1 Main GO ontologies identified in secretome samples. The pictures depict some common ontologies identified by PANTHER analysis in the secretomes of VWAT-MSCS, sWAT-MSCS, and BM-MSCs. In orange are factors classified according GO Biological activity and GO Pathway, while in blue are classified according GO Cellular component, GO Protein class and GO molecular function

and SCF-beta-TrCP mediated degradation of Emi1. These three networks are associated with the identified GO terms that are present in all secretomes coming from MSCs of ND-treated mice. For example, within the ontologies associated with endoplasmic reticulum stress (Table 3, Fig. 1), the most significant network is the endosome pathway leading to antigen processing (Table 4). In vWAT-MSC secretomes, the Reactome analysis identified 14 proteins out of 51 in the reference list. In sWAT-MSC and BM-MSC secretomes, 17 and 14 proteins belonging to this network, respectively, were present (Fig. 2; Additional file 4).

The most significant network in protein anabolism/catabolism ontologies (Fig. 1) is the post-translational protein phosphorylation (Table 4; Additional file 4).

The Reactome pathway "SCF-beta-TrCP mediated degradation of Emi1" indicates Emil protein destruction in early mitosis by the SCF $\beta \operatorname{TrCP} /$ Slimb Ubiquitin Ligase, which activates the anaphase-promoting complex to allow cell cycle progression [19]. This network cannot be assigned to a single GO entity; rather it refers to several ontologies associated with cell signaling (Tables 2 and 3).

Several Reactome pathways specifically identified in the vWAT-MSC secretome can be associated with protein anabolism/catabolism GO terms, including: formation of a pool of free $40 \mathrm{~S}$ subunits; peptide chain elongation; and eukaryotic translation termination
(Table 4). Selenocysteine synthesis appears to be the most significant pathway that could be associated with the oxy-redox GO terms. Many other pathways involved in cell cycle regulation were found in the vWAT-MSC secretome besides the SCF-beta-TrCP mediated degradation of Emi1 that was in common with other secretomes. Notably, Reactome analysis identified a pathway named platelet degranulation, which can refer to several GO terms listed in Tables 3 and 4 (Fig. 3). Activated platelets rapidly release the contents of distinct types of preformed intracellular vesicles (granules), such as dense granules, alpha granules, and lysosomes. Dense granule components contribute to hemostasis and coagulation, but they also play a role in cancer metastasis. Alpha granules contain cytokines, growth factors, regulators of the coagulation cascade, pro- and anti-inflammatory factors, and other bioactive factors that contribute to a number of disease processes [20].

In the sWAT-MSC secretome, several pathways are associated with cytoskeleton and ECM GO ontologies, including: crosslinking of collagen fibrils; laminin interactions; and anchoring fibril formation (Table 4). Additionally, the BM-MSC cells release factors that belong to pathways related to cytoskeleton and ECM organization (Table 4). In addition, the secretome of BM-MSCs contain proteins belonging to the platelet degranulation pathway, as reported for the vWAT-MSC 


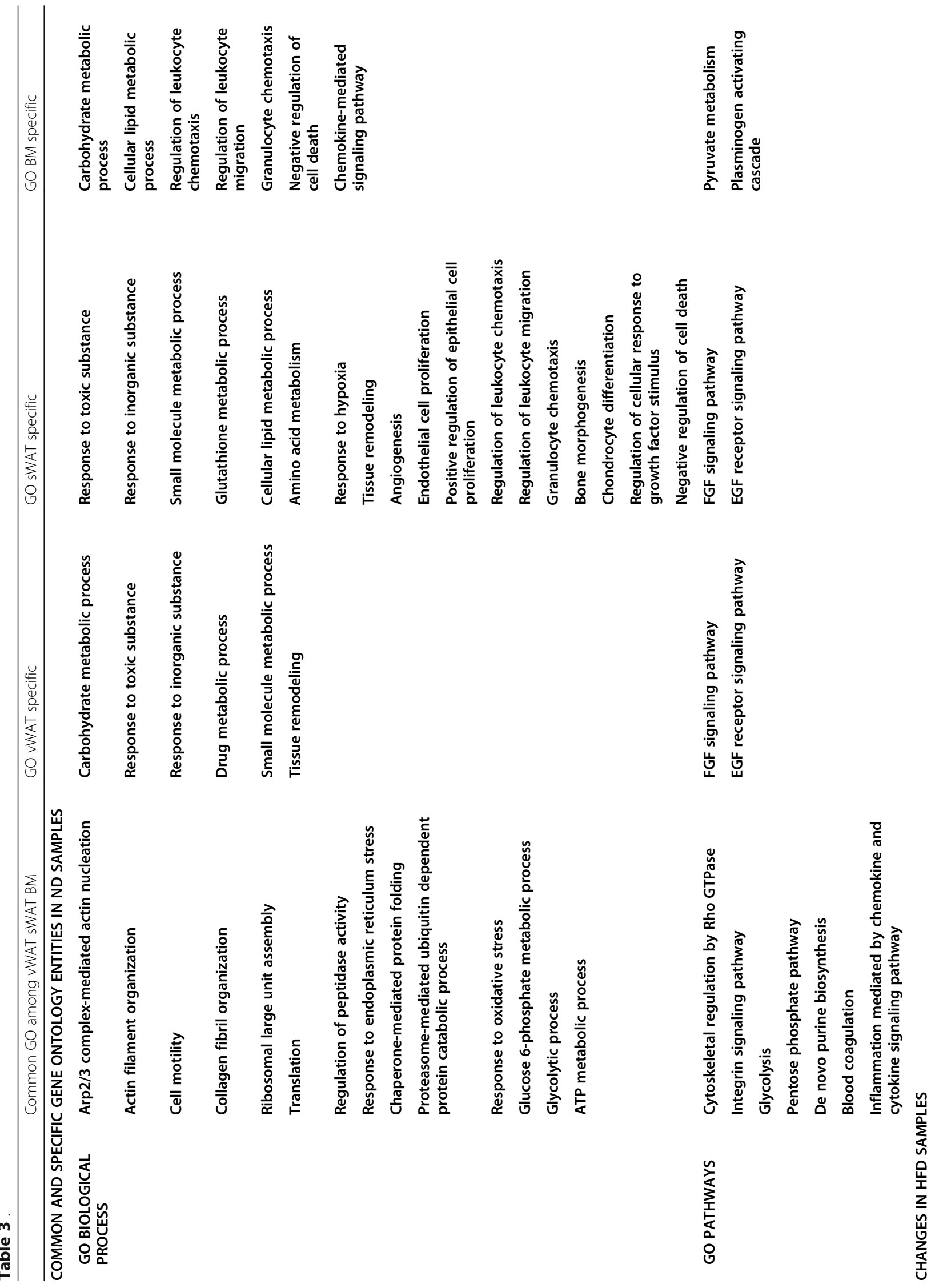




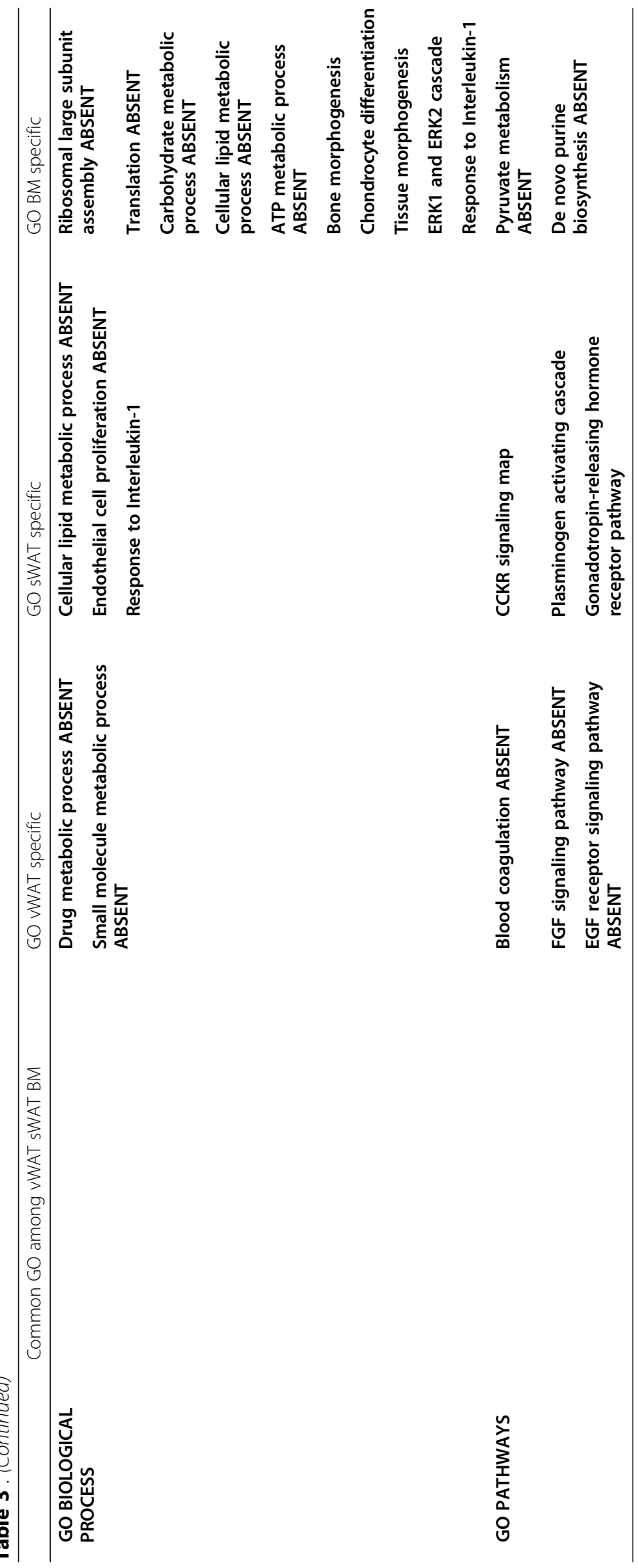


Table 4 .

vWAT ND REACTOME PATHS (27)

$\mathrm{APC} / \mathrm{C}: \mathrm{Cdc} 20$ mediated degradation of Securin

APC/C:Cdh1 mediated degradation of $\mathrm{Cdc} 20$ and other APC/C:Cdh1 targeted proteins in late mitosis/early $\mathrm{G} 1$

Autodegradation of Cdh1 by Cdh1:APC/C

CDK-mediated phosphorylation and removal of Cdc6

CDT1 association with the CDC6:ORC:origin complex

Chk1/Chk2(Cds1) mediated inactivation of Cyclin B:Cdk1 complex

Cross-presentation of soluble exogenous antigens (endosomes)

Defective CFTR causes cystic fibrosis

Degradation of AXIN

Eukaryotic Translation Termination

Formation of a pool of free $40 \mathrm{~S}$ subunits

Hh mutants abrogate ligand secretion

Hh mutants that don't undergo autocatalytic processing are degraded by ERAD

HSF1 activation

L13a-mediated translational silencing of Ceruloplasmin expression

Mycobacterium tubercolisis biological processes

Orc1 removal from chromatin

Peptide chain elongation

Platelet degranulation

Post-translational protein phosphorylation

Regulation of activated PAK-2p34 by proteasome mediated degradation

Regulation of ornithine decarboxylase (ODC)

Regulation of RAS by GAPs

Response to elevated platelet cytosolic $\mathrm{Ca} 2+$

SCF-beta-TrCP mediated degradation of Emi1

Selenocysteine synthesis

Vif-mediated degradation of APOBEC3G

sWAT ND REACTOME PATHS (13)

Anchoring fibril formation

Assembly of collagen fibrils and other multimeric structures

Cross-presentation of soluble exogenous antigens (endosomes)

Crosslinking of collagen fibrils

Elastic fibre formation

Hh mutants that don't undergo autocatalytic processing are degraded by ERAD

Laminin interactions

Mycobacterium tuberculosis biological processes

Post-translational protein phosphorylation

Prefoldin mediated transfer of substrate to $\mathrm{CCT} / \mathrm{TriC}$

Regulation of ornithine decarboxylase (ODC)

SCF-beta-TrCP mediated degradation of Emi1

Vif-mediated degradation of APOBEC3G

BM ND REACT PATHS (17)

Assembly of collagen fibrils and other multimeric structures 
Table 4 . (Continued)

Collagen chain trimerization

Collagen degradation

Cross-presentation of soluble exogenous antigens (endosomes)

Crosslinking of collagen fibrils

Defective B4GALT1 causes B4GALT1-CDG (CDG-2d)

Defective CHST6 causes MCDC1

Defective ST3GAL3 causes MCT12 and EIEE15

Diseases associated with glycosaminoglycan metabolism

ECM proteoglycans

Elastic fibre formation

Laminin interactions

Molecules associated with elastic fibres

Platelet degranulation

Post-translational protein phosphorylation

Regulation of Insulin-like Growth Factor (IGF) transport and uptake by Insulin-like Growth Factor Binding Proteins (IGFBPs)

SCF-beta-TrCP mediated degradation of Emi1

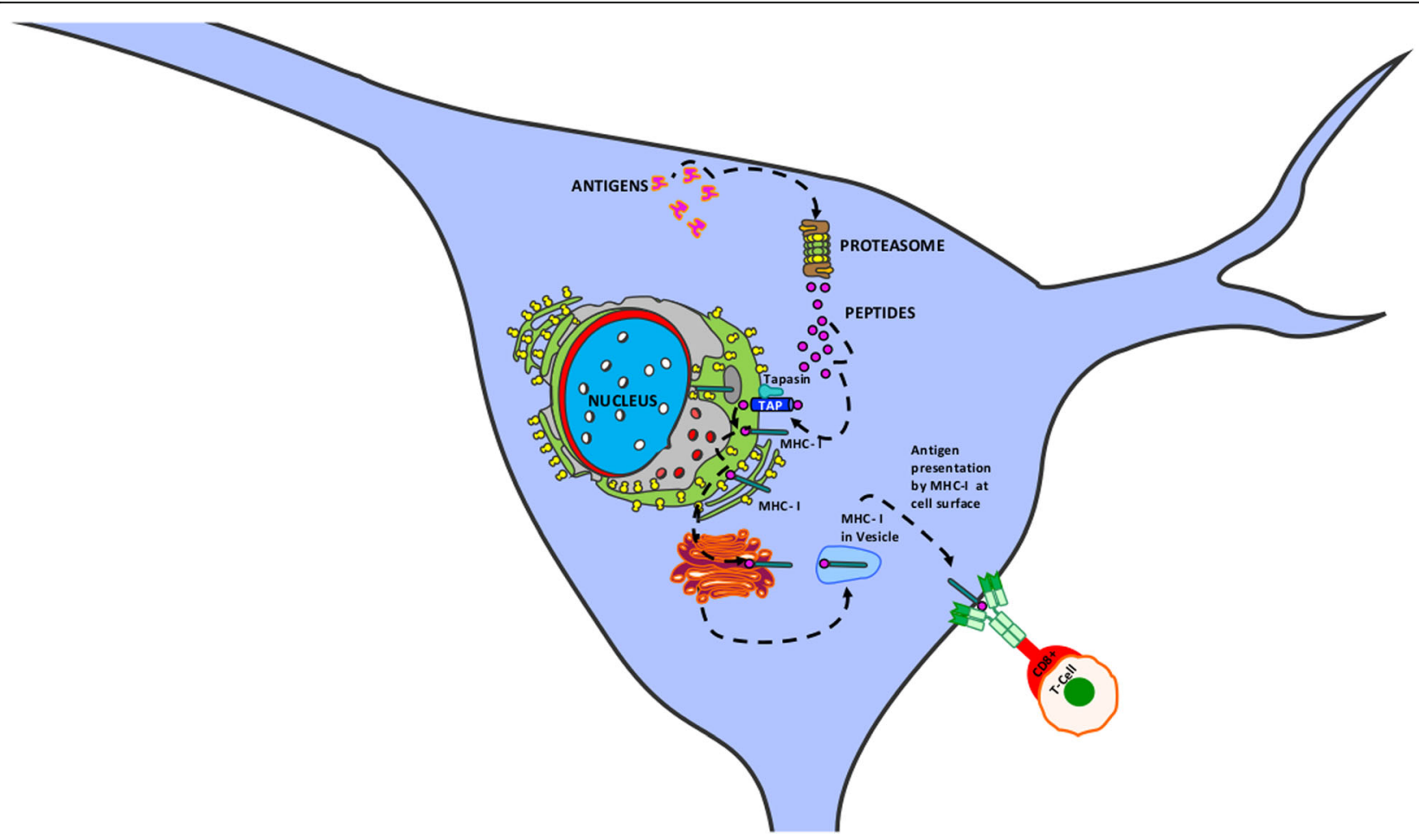

Fig. 2 Cross-presentation of soluble exogenous antigens (endosomes) pathway. The pathway consists of three main networks: antigen processing — cross-presentation; antigen presentation—folding, assembly, and peptide loading of class I MHC; and antigen

processing —ubiquitination and proteasome degradation. During the presentation process, antigen proteins are degraded into peptides by proteases in the proteasome. Peptides are then delivered to the endoplasmic reticulum (ER) through heat shock proteins and the transporter associated with antigen processing (TAP), which transport peptides from cytosol into the ER lumen. Several ER chaperones (calnexin, tapasin, calreticulin, etc.) contribute to MHC-I assembly. Peptides are loaded into the MHC-I peptide binding groove; this complex exits the ER and is transported to Golgi and then to the cell surface by exocytic vesicles. Naiive T cells (CD8+) are activated by interacting with peptide-MHC-I complexes. Additional file 4 reports the proteins of VWAT-MSC, sWAT-MSC, and BM-MSC secretomes that belong to the above-indicated networks 


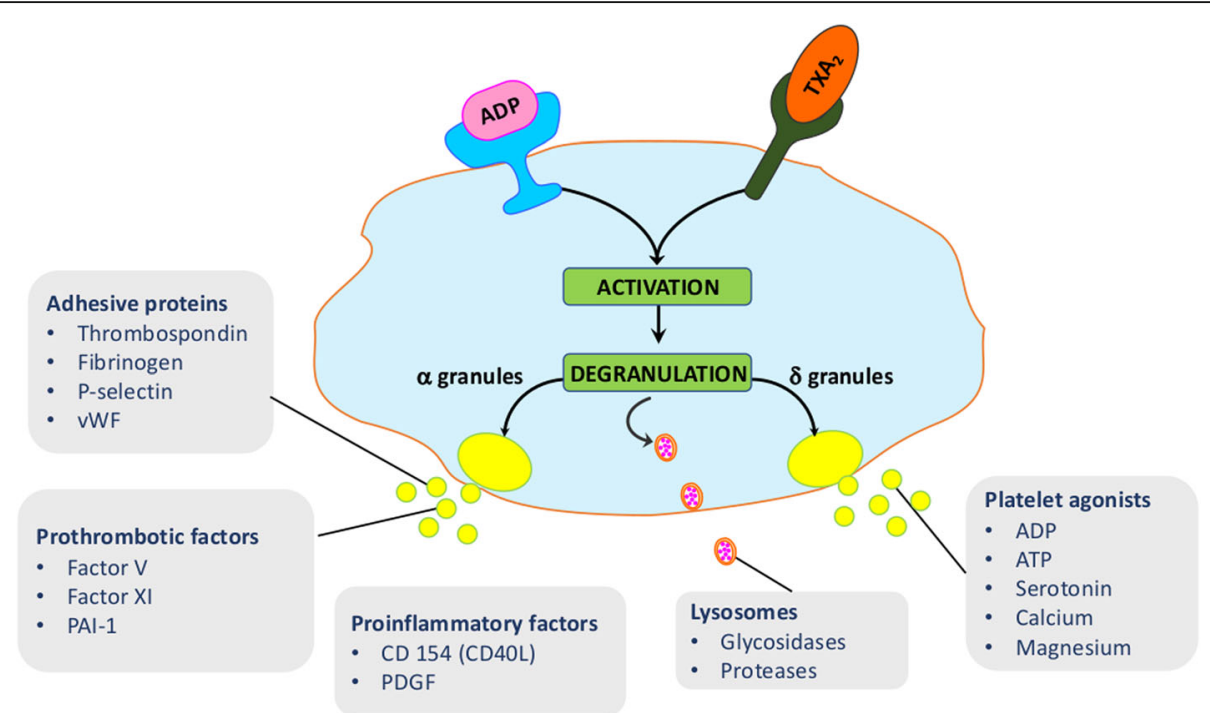

Fig. 3 Platelet degranulation pathway. This pathway consists of several networks: ABCC4 accumulation of dense granule contents; exocytosis of platelet dense granule content; surface deployment of platelet dense granule membrane components; exocytosis of platelet alpha granule contents; surface deployment of platelet alpha granule membrane components; release of platelet cytosolic components; release of platelet secretory granule components; and exocytosis of proactivator polypeptide. Platelets are activated following the interaction between ligands, such as ADP and TXA2 (Tromboxane A2), and their cognate receptors on the platelet cell surface. After activation, platelets release the contents of three distinct types of preformed intracellular vesicles. Dense granules ( $\delta$ granules) contain platelet agonists, and lysosomes contain glycosidases and acid proteases. The a granules release adhesive proteins, prothrombotic factors, and pro-inflammatory factors. Additional file 4 reports the proteins of VWAT-MSC, sWAT-MSC, and BM-MSC secretomes that belong to these networks

secretome. Regulation of the insulin-like growth factor pathway is a peculiar network identified in the secretome of BM-MSCs (Fig. 4).

\section{Reactome analysis in samples from HFD-treated mice}

The secretome contents of vWAT-MSCs, sWAT-MSCs, and BM-MSCs obtained from obese mice were assigned to 25,15 and 20 Reactome pathways, respectively (Table 5). Most of the Reactome pathways found in the corresponding secretomes obtained from normal mice were also present in samples from obese mice. In particular, the three pathways that were in common among the secretomes of sWAT-MSCs, vWAT-MSCs, and BMMSCs in normal mice were also identified in obese mice.

A deep examination into the secretome of vWATMSCs shows that the selenocysteine synthesis pathway present in samples from normal mice was absent in samples coming from obese mice.

The sWAT-MSCs of HFD-treated samples secreted proteins belonging to the platelet degranulation pathway that were absent in the corresponding ND-treated samples. Thus, in obese mice, all three types of MSCs release factors activating platelets. This overproduction of platelet-activating factors may contribute to the chronic inflammation associated with obesity. The release of proteins belonging to the neutrophil degranulation pathway from BM-MSCs, seen in obese mice, could further exacerbate inflammation.
Identification of proteins specifically expressed in samples from ND- and HFD-treated mice

We performed a Venn diagram analysis to identify common and specific proteins in the different environmental and pathological conditions. The MSCs isolated from different tissues in normal mice released only partially overlapping factors (Fig. 5). Specifically, 64 proteins were found exclusively in the secretome of vWAT-MSCs, while 144 and 69 were exclusively present in the secretomes of sWAT-MSCs and BM-MSCs, respectively. Additionally, in obese mice, MSCs from different sources shared only part of their secretomes.

We then compared the proteins exclusively present in vWAT-MSCs between normal and obese mice. The pathological condition greatly affected the secretome composition: only 7 proteins were found both in normal and obese secretome samples, while 57 were exclusively present in the secretome of normal samples and 29 were exclusively present in the secretome of obese samples (Fig. 5). The secretomes of sWAT-MSCs and BM-MSCs were also greatly modified by obesity (Fig. 5).

We then focused on proteins exclusively released by vWAT-MSCs, sWAT-MSCs, or BM-MSCs isolated from samples taken from normal and obese mice (Table 6, Additional file 2). The most significant proteins released exclusively from the vWAT-MSCs of normal mice belong to several networks. For example, Ptgr1 and Csfr1 are part of the modulation of the immune system. Ptgr1 


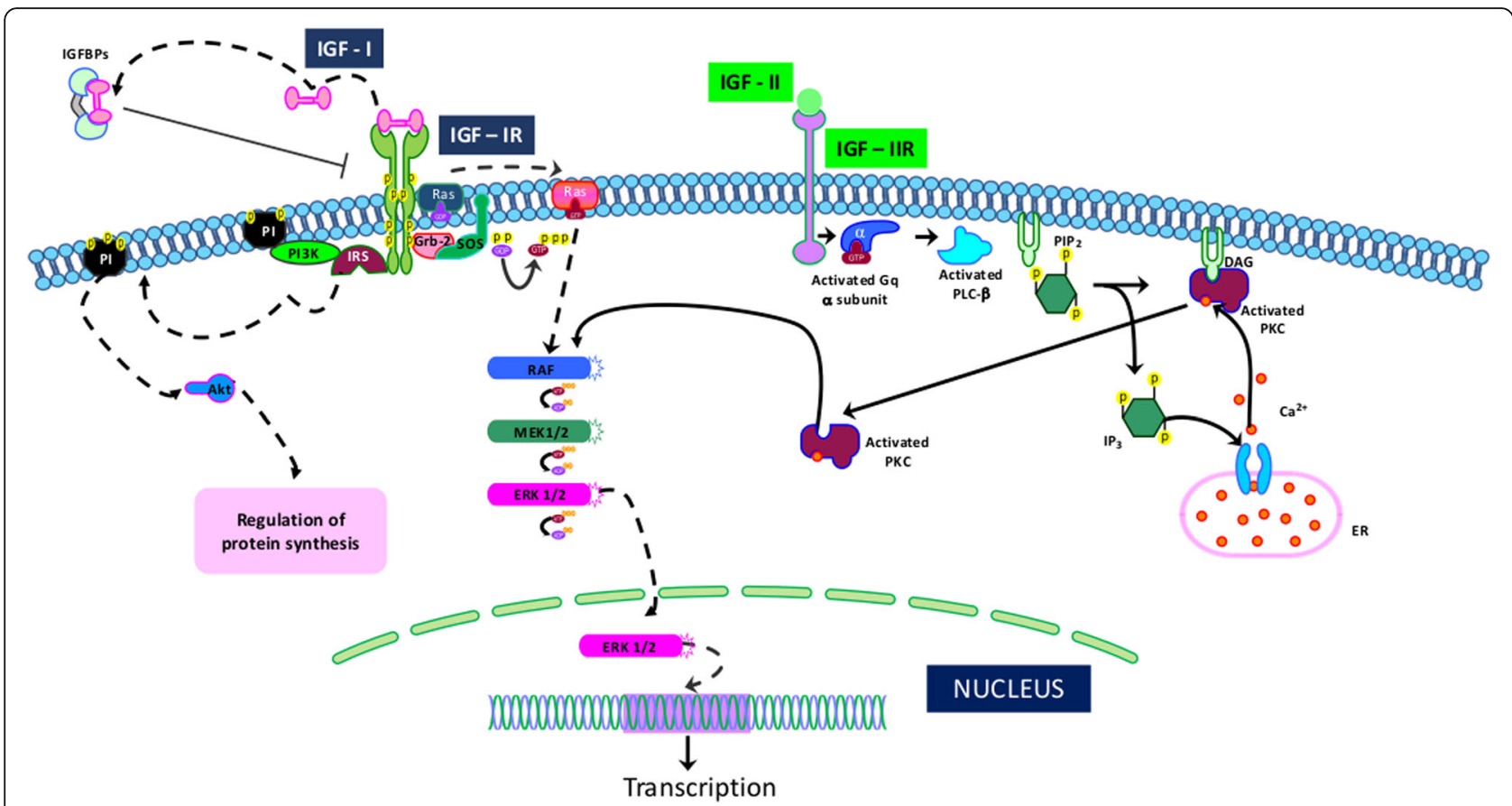

Fig. 4 Regulation of insulin-like growth factor (IGF) transport and uptake by insulin-like growth factor binding proteins (IGFBPs) pathway. The pathway consists of several networks: IGFBP1 binds with IGF, forming IGF:IGFBP1; IGFBP2 binds with IGF, forming IGF:IGFBP2; IGFBP4 binds with IGF, forming IGF:IGFBP4; IGFBP6 binds with IGF, forming IGF:IGFBP6; PAAP-A proteolyzes IGF:IGFBP4; FAM20C phosphorylates FAM20C substrates. IGF-I binds to its receptor (IGF-IR), which leads to IRS/PI3K phosphorylation and subsequent downstream activation of AKT. Alternatively, IGF-I can activate Shc/Grb-2/Sos phosphorylation and complex formation. This event promotes the activation of the Ras/Raf/MEK/MAPK cascade. IGF-I binds to the hybrid IGF-IR/IR receptor, activating PI3K and MAPK pathways. The IGF-II/IGF-IIR complex can activate an alternative pathway that is associated with the G protein and phospholipase C (PLC). The result of the PLC activity is the production of diacylglycerol (DAG) and inositol triphosphate (IP3), which in turn can activate protein kinase C (PKC) and the RAF/MEK/ERK pathway. IGF-I also binds with IGF-IIR, and IGF-II also binds with IGF-IR. It not well-known which pathways are activated following these interactions. IGFBP proteins bind with either IGF-I or IGF-II and modulate their activities

is involved in a key step of the metabolic inactivation of leukotriene B4, whose levels increase during inflammation [21]. Csfr1 signaling is fundamental to the differentiation and survival of the mononuclear phagocyte system and macrophages [22]. Catalase and GSR are components of the redox activity network. Catalase protects cells from the toxic effects of hydrogen peroxide, and GSR maintains high levels of reduced glutathione in the cell cytoplasm [23]. BLVRA, CRAT, Nampt, and Sorcin are part of metabolic networks. BLVRA reduces biliverdin (a byproduct of heme catabolism) to bilirubin, which is an antioxidant and has a role in lowering risk of metabolic syndromes. Obese individuals with high visceral adiposity have low bilirubin levels [24]. CRAT-i.e., carnitine acetyltransferase-is a mitochondrial enzyme that catalyzes the interconversion of acetylcarnitine and acetyl-CoA. Studies have shown that it is a positive regulator of total body glucose tolerance and muscle activity, and its activity is inhibited by obesity and lipid stress [25]. Nampt, also called Visfatin, is an adipokine that influences metabolic homeostasis and whose level increases significantly with obesity, due to increased body mass index [26]. Sorcin is a protein involved in maintaining calcium within the endoplasmic reticulum by inhibiting ryanodine receptor activity; its impairment is associated with metabolic syndromes [27].

All of these proteins have a positive role in several aspects of organismal homeostasis, and their presence is lost in the secretomes of vWAT-MSCs in samples taken from obese mice.

The most significant proteins released exclusively from sWAT-MSCs from normal mice belong to the following networks: redox activity, modulation of immune system, growth factor activities, and differentiation network (Table 6). Ang, Fstl3, Pgf, and Angptl4 are part of this last network. Ang (angiogenin), Pgf (placenta growth factor), and Angptl4 (angiopoietin-like 4) could be the key players in angiogenesis of the sWAT-MSC secretome, as evidenced in the Reactome analysis [28-30]. Fstl3 (follistatin) may be one the most important components of the sWAT-MSC secretome, since it conducts key functions in regulation of fat accumulation and insulin sensitivity, modulation of hematopoiesis, and control of bone formation [31-33]. The GCL, Prdx5, and Prdx6 
Table 5 .

vWAT HFD REACTOME PATHS (25)

Anchoring fibril formation

APC/C:Cdc20 mediated degradation of Securin

APC/C:Cdh1 mediated degradation of Cdc20 and other APC/C:Cdh1 targeted proteins in late mitosis/early G1

Assembly of collagen fibrils and other multimeric structures

Autodegradation of Cdh1 by Cdh1:APC/C

CDK-mediated phosphorylation and removal of $\mathrm{Cdc} 6$

CDT1 association with the CDC6:ORC:origin complex

Chk1/Chk2(Cds1) mediated inactivation of Cyclin B:Cdk1 complex

Collagen chain trimerization

Collagen degradation

Cross-presentation of soluble exogenous antigens (endosomes)

Crosslinking of collagen fibrils

Defective CFTR causes cystic fibrosis

Degradation of AXIN

Hh mutants abrogate ligand secretion

Hh mutants that don't undergo autocatalytic processing are degraded by ERAD

HSF1 activation

Orc1 removal from chromatin

Platelet degranulation

Post-translational protein phosphorylation

Regulation of activated PAK-2p34 by proteasome mediated degradation

Regulation of ornithine decarboxylase (ODC)

Regulation of RAS by GAPs

SCF-beta-TrCP mediated degradation of Emi1

Vif-mediated degradation of APOBEC3G

sWAT HFD REACT PATHS (15)

Assembly of collagen fibrils and other multimeric structures

Autodegradation of $\mathrm{Cdh} 1$ by $\mathrm{Cdh} 1: \mathrm{APC} / \mathrm{C}$

Cross-presentation of soluble exogenous antigens (endosomes)

Crosslinking of collagen fibrils

Defective B4GALT1 causes B4GALT1-CDG (CDG-2d)

Elastic fibre formation

Hh mutants abrogate ligand secretion

Hh mutants that don't undergo autocatalytic processing are degraded by ERAD

Laminin interactions

Mycobacterium tuberculosis biological processes

Platelet degranulation

Post-translational protein phosphorylation

Regulation of ornithine decarboxylase (ODC)

SCF-beta-TrCP mediated degradation of Emi1

Vif-mediated degradation of APOBEC3G

BM HFD REACT PATHS (20)

Anchoring fibril formation

Assembly of collagen fibrils and other multimeric structures 
Table 5 . (Continued)

Collagen biosynthesis and modifying enzymes
Collagen chain trimerization
Collagen degradation
Collagen formation
Cross-presentation of soluble exogenous antigens (endosomes)
Crosslinking of collagen fibrils
Defective B4GALT1 causes B4GALT1-CDG (CDG-2d)
Degradation of the extracellular matrix
ECM proteoglycans
Elastic fibre formation
HSF1 activation
Laminin interactions
Molecules associated with elastic fibres
NCAM1 interactions
Neutrophil degranulation
Platelet degranulation
Post-translational protein phosphorylation
Regulation of Insulin-like Growth Factor (IGF) transport and uptake by Insulin-like Growth Factor Binding Proteins (IGFBPs)

proteins are part of the redox activity network. GCL (glutamate cysteine ligase) is an enzyme of the cellular glutathione biosynthetic pathway; together with Prdx 5 and Prdx6, it is fundamental in controlling reactive oxygen levels and in counteracting oxidative stress [34, 35].
The tissue development and differentiation functions-along with the anti-oxidant activity present in the secretome of sWAT-MSCs from normal mice-are absent in samples from obese mice. Instead, in the secretomes from obese mice, factors are present whose activities are strictly associated with negative outputs of

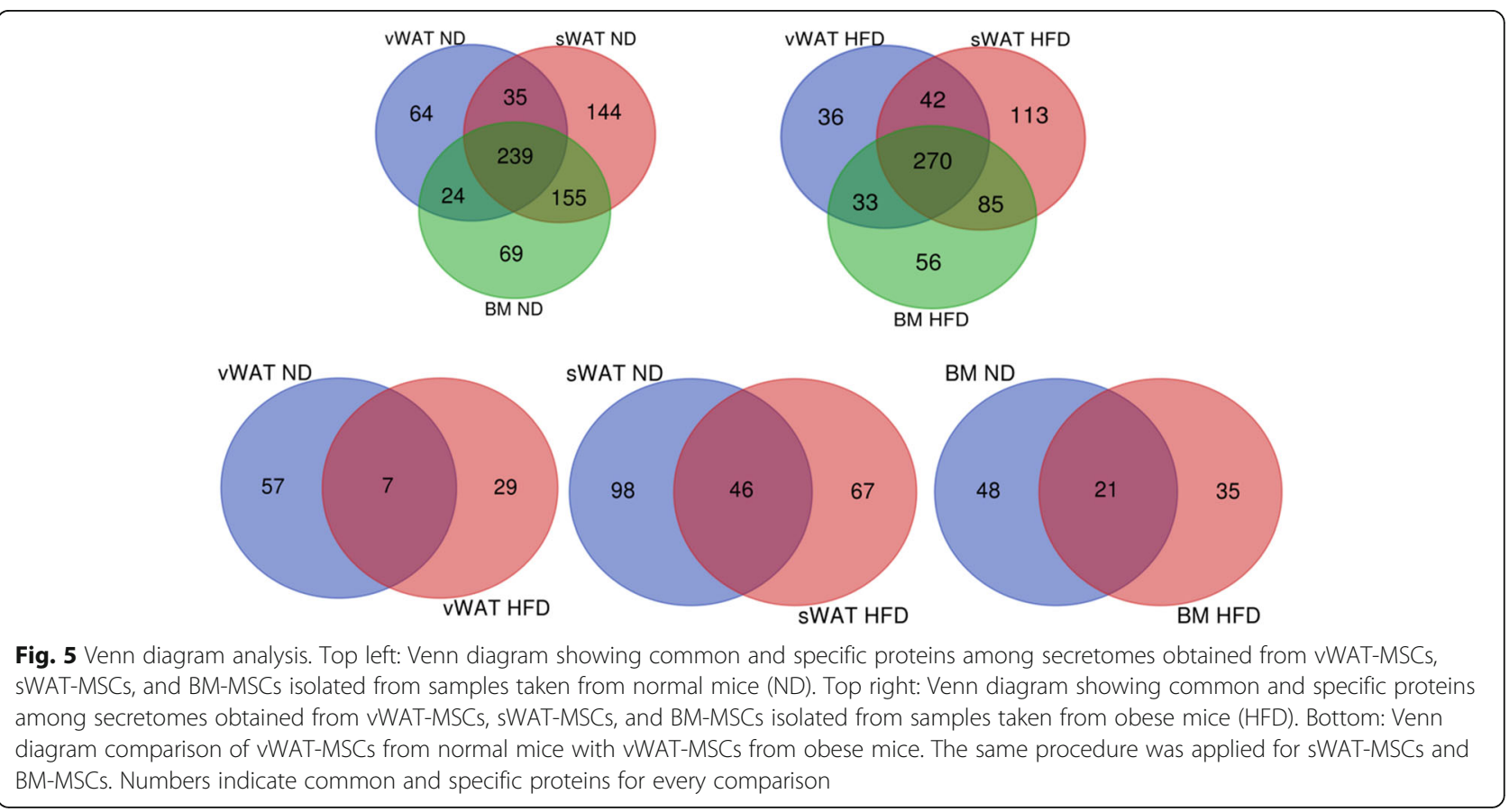


Table 6 Proteins specifically expressed in the indicated secretomes

\begin{tabular}{|c|c|c|c|}
\hline & vWAT ND & sWAT ND & BM ND \\
\hline \multirow[t]{4}{*}{ Growth factor activity and differentiation } & & Ang & Gmfb \\
\hline & & Angpt/4 & Manf \\
\hline & & Fstl3 & \\
\hline & & Pgf & \\
\hline \multirow[t]{2}{*}{ Modulation of immune system } & Ptgr1 & $\mathrm{Cd} 81$ & Ccl9 \\
\hline & Csfr1 & & Ifi30 \\
\hline \multirow[t]{3}{*}{ Redox activity } & Catalase & Glc & \\
\hline & Gsr & Prdx5 & \\
\hline & & Prdx6 & \\
\hline \multirow[t]{4}{*}{ Metabolism } & Blvra & & Aldh1a3 \\
\hline & Crat & & Aldh1a2 \\
\hline & Nampt & & Me1 \\
\hline & Sorcin & & \\
\hline \multirow[t]{4}{*}{ ECM } & & & Cemip \\
\hline & & & Itih3 \\
\hline & & & Vcan \\
\hline & vWAT HFD & sWAT HFD & BM HFD \\
\hline \multirow[t]{3}{*}{ Growth factor activity and differentiation } & Hdgf & $\operatorname{lgf} 2$ & Fstl3 \\
\hline & & Ostf1 & \\
\hline & & Tgm2 & \\
\hline Modulation of immune system & & & Cfh \\
\hline \multicolumn{4}{|l|}{ Redox activity } \\
\hline \multirow[t]{2}{*}{ Metabolism } & & Fdps & Lipa \\
\hline & & Pla1a & \\
\hline \multirow[t]{2}{*}{ Miscellaneous/pathological conditions } & & Hyou1 & \\
\hline & & Mt1 & \\
\hline
\end{tabular}

obesity. For example, Ostf1 (osteoclast stimulation factor 1) can promote osteoporosis, Tgm 2 is involved in negative artery remodeling, and IGF2 can contribute to senescence of MSCs [36-38].

BM-MSCs release factors involved in growth and differentiation of neural cells, such as glia maturation factor- $\beta$ (GMFB) and mesencephalic astrocyte-derived neurotrophic factor (MANF) [39, 40]. These cells also release proteins that regulate energy metabolism, such as Me1 (malic enzyme), Aldh1a2, and Aldh1a3 (aldehyde dehydrogenase) [41, 42]. BM-MSCs also secrete many proteins associated with glycosaminoglycan formation and degradation. All these factors were absent in the secretomes of cells isolated from tissue samples of obese mice.

\section{Discussion}

Release of signaling factors is a key activity of MSCs; for this reason, several studies have analyzed their secretome content. Nevertheless, a systemic investigation of the microenvironment's influence on MSC secretome composition, either in physiological or pathological conditions, is still lacking. Indeed, the microenvironmentwith structural and trophic support, topographical information, and pathophysiological cues-can greatly affect cell behavior [43].

The literature contains findings that address specific aspects of MSC secretome. For example, some researchers have analyzed the cytokines released by adipose tissue-derived and bone marrow-derived MSCs, while others have focused their attention on secreted neuroregulators or on factors involved in hepatic lineage development and differentiation [8, 44, 45]. Some researchers have analyzed the contents of extracellular vesicles released by adipose tissue-derived MSCs $[8,46]$. Others have performed secretome analysis with lowresolution techniques, which has not provided exhaustive information $[47,48]$.

Our study aimed to fill certain gaps in secretome analysis of MSCs by performing a comparison analysis of 
the impact of physiological (tissue of origin) and pathological (obesity) cues. The choice to analyze MSCs from visceral WAT and subcutaneous WAT was not trivial, since these tissues have distinct metabolic and inflammatory functions [49]. Indeed, the vast majority of studies have analyzed the biological properties of MSCs derived from subcutaneous fat, and only a few have analyzed those derived from visceral fat. However, the latter fat depot contributes remarkably to the negative effects of obesity on human health. In this context, we evaluated the effect of obesity on MSC secretory activity, since this condition affects the size, function, and inflammatory state of adipose tissues and modifies the stem cell niches present in these tissues [12, 49].

Our study clearly showed that tissue microenvironment significantly affects secretome composition of MSCs and hence their signaling activity. First, it should be emphasized that most of the proteins found in the MSC secretomes lack the signal peptide present at the $\mathrm{N}$-terminus of many proteins that are destined for the secretory pathway [50]. This suggests that many of them are not freely circulating within extracellular fluids but are rather encapsulated in EVs.

The MSCs isolated from bone marrow, visceral WAT, and subcutaneous WAT of healthy mice share a common core of released factors: components of cytoskeletal and extracellular structures; regulators of basic cellular functions, such as protein synthesis and degradation; modulators of endoplasmic reticulum stress; and counteracting oxidative stress. It can be hypothesized that MSC secretome beneficially affects target cells by contributing to their main biological activities through EVmediated horizontal transfer of structural cellular components and of regulators of cellular anabolism and catabolism processes.

However, each type of MSCs may exert specific signaling functions, which could be determined by looking at the many factors that are exclusively released from each MSC type. The vWAT-MSCs release factors that have a peculiar role in detoxification activity in response to toxic substances and drugs as well as many factors involved in the synthesis of selenocysteine, which is present in the active sites of several enzymes (glutathione peroxidase, thioredoxin reductase, and iodothyronine deiodinase) that participate in oxidation-reduction reactions [51]. These functions of MSCs within vWAT could have a potential role in preserving the tissue's healthiness, since many findings have demonstrated that adipose tissue is a potential site of reactive oxygen species (ROS) and toxin accumulation [52]. Obesity status almost completely negated the release of these adipose tissue "protective factors".

The sWAT-MSC secretome contains many proteins involved in tissue development and differentiation, such as factors participating in chondrogenesis, osteogenesis, and angiogenesis. This last process seems to be highly supported by sWAT-MSC signaling, since these cells released angiogenin, placenta growth factor, and Angptl4, which have a prominent role in angiogenic processes [28-30]. At the same time, we find only a few factors involved in adipogenesis [53]. This may indicate that their levels are below the limit of detection for our technique and/or that MSCs are not the main producers of such factors. It is well-known that MSCs play a key role in immunomodulation; our study demonstrated that the sWAT-MSCs release many proteins involved in chemotaxis and migration of immune cells. Obesity negatively impacted sWAT-MSC secretome: the anti-oxidant (GCL, Prdx5, Prdx6) and tissue development (Ang, Angptl4, Fstl3, Pgf) activities were lost, while factors promoting osteoporosis and negative vessel remodeling were acquired.

The analysis of BM-MSC secretome in tissue from normal mice revealed that these cells exert a signaling function through a very active remodeling of extracellular matrix structures; factors (CEMIP, Itih3, VCAN) that reshape (build/degrade) glycosaminoglycans were only present in their secretome. These cells also seemed to play a role in metabolism control by releasing dozen of factors, some of them found exclusively in their secretome (Aldh1a3, Aldh1a2, Me1). Of great interest, in BM-MSC secretome includes factors that promote growth and differentiation of glia and neurons, such as glia maturation factor- $\beta$ (GMFB) and mesencephalic astrocyte-derived neurotrophic factor (MANF) $[39,40]$. The presence of such factors matches the hypothesized crosstalk between osteogenic and neurogenic niches, which relies on partial overlap of the molecular and secretome profiles as well as on the intimate relationship with vessels [54]. At the same time, the trophic effects of GMFB and MANF apply not only to neurons and glia but also to other cell types [40, 55].

How does a pathological modification of tissue microenvironment affect the secretome composition of MSCs? Obesity, with its associated chronic inflammation status, profoundly modifies the secretome content of MSCs. Obesity status almost completely negated the release of factors that promote tissue renewal and homeostasis. In obese mice, vWAT-MSCs lost their particular detoxification and ROS scavenging functions. Anti-oxidant activities were also impaired in the secretomes of sWATMSCs and BM-MSCs. This occurrence could negatively impact the health of obese individuals. High-caloric intake produces an excess of energy substrates for cellular metabolic pathways, which in turn increase ROS production that cannot be buffered. In obese individuals, the ROS increment alters cellular functions and may induce cellular senescence, as shown by us and other 
researchers. Obesity negatively impacted the sWAT-MSC secretome, since its anti-oxidant (GCL, Prdx5, Prdx6) and tissue development (Ang, Angptl4, Fstl3, Pgf) activities were lost, while factors promoting osteoporosis and negative vessel remodeling were acquired. These events were associated with secretion of pro-inflammatory cytokines, associated with the IL-1 signaling pathway and platelet degranulation. The release of inflammatory factors belonging to these pathways was also detected in the BM-MSCs secretome in obese mice, along with cytokines promoting neutrophil degranulation.

\section{Conclusion}

We demonstrated that the content of MSC secretomes depends on tissue microenvironment and that pathological condition may profoundly alter its composition. This study demonstrates that MSCs isolated from different tissues both share common functions and perform unique tasks. This finding may pave the way to better understanding the role of MSCs in tissue renewal and homeostasis. Moreover, it may further contribute to selection of the correct MSC source(s) for clinical purposes. In cell therapy treatments, the choice of adipose tissue-derived MSCs or bone marrow-derived MSCs is not irrelevant and may have profound consequences on the clinical outcomes.

\section{Supplementary information}

Supplementary information accompanies this paper at https://doi.org/10. 1186/s12964-020-00614-w.

Additional file 1 Flow cytometry analysis and differentiation of MSCs. Panel A: BM-MSCs, VWAT-MSCs, and sWAT-MSCs were trypsinized; washed with PBS; and incubated with either anti-CD105 FITC (sc-18,893 FITC), anti-CD90 PE (SC-1914 PE), or anti-CD73 PE (sc-398,260). The antibodies were used according to the manufacturer's procedures (Santa Cruz Biotechnology (A, USA). After 30 min of incubation with the antibodies at room temperature, cells were washed with PBS and resuspended in FACS buffer on a Guava EasyCyte flow cytometer for data acquisition (Merck Millipore MA, USA). We performed data analysis with a standard procedure using EasyCyte software. A minimum of 5000 cells per sample were analyzed and gated for forward scatter (FSC) versus side scatter (SSC) channel signals. In each panel, the histograms show positive cells from obese (blue) and normal (green) mice. ND = Normal Diet-treated mice; $\mathrm{HFD}=$ High Fat Diet-treated mice. In red are the negative controls. Panel B: Adipocyte, osteocyte, and chondrocyte differentiation of MSCs obtained from obese and normal mice. The figure shows representative images of Oil Red O (adipocytes), Alizarin Red S (osteocytes), and Alcian blue (chondrocytes) staining for every experimental condition. The method for differentiation is described below. Adipogenic differentiation. MSCs were treated for 15 days in a mesenchymal stem cell adipogenic differentiation medium (PT-3004-KT; Lonza, Walkersville, MD, USA). The medium contained insulin (recombinant), dexamethasone, indomethacin, and 3-isobuty-l-methyl-xanthine (IBMX). Lipid droplets were revealed by staining with Oil Red O. Osteogenic differentiation. MSCs were treated for 15 days in a mesenchymal stem cell osteogenic differentiation medium (PT-3002-KT; Lonza). The medium contained dexamethasone, ascorbate, and glycerophosphate. Staining with Alizarin Red S revealed calcium deposits in differentiated osteocytes. Chondrogenic differentiation. MSCs were seeded as pellets in 96 round-bottom multi-wells and cultured in a chondrogenic medium composed of DMEM, 1\% FBS, 50 nM ascorbate-2- phosphate (Sigma-Aldrich, St. Louis, MO, USA), $0.1 \mathrm{mM}$ dexamethasone (Sigma-Aldrich, MO, USA), and $10 \mathrm{ng} / \mathrm{mL}$ human transforming growth factor (hTGF)- $\beta 1$ (PeproTech, London, UK). After 21 days, Alcian blue staining was performed.

Additional file 2. List of proteins identified in MSC secretome. "ND HFD tech biol replicates" spreadsheet: The sheet shows the list of proteins found in VWAT-MSCS, sWAT-MSCs, and BM-MSCs isolated from samples taken from ND-treated mice designated as 1, 2, and 3 and from HFDtreated mice designated as 4, 5, and 6. For each biological sample, there were two technical replicates (A, B). Proteins were listed with their UniProt identifiers. "ND HFD common data" spreadsheet: The proteins secreted by VWAT-MSCs isolated from samples taken from mouse 1, 2, and 3 were analyzed with a Venn graph to find common data. The procedure was also performed for sWAT-MSCs and BM-MSCs. The sheet also lists proteins isolated from samples taken from mice 4, 5, and 6, which were analyzed with the same method. "Venn comparison in ND or HFD" spreadsheet: The sheet shows the result of Venn diagram comparison among VWAT-MSCs, sWAT-MSCs, and BM-MSCs coming from ND- and HFD-treated mice. "Venn comparison in ND vs. HFD" spreadsheet: The sheet shows the result of Venn diagram comparison of VWAT-MSCs from ND-treated mice versus VWAT-MSCs from HFD-treated mice. The same procedure was employed for sWAT-MSCs and BM-MSCs.

Additional file 3. GO analysis carried out with PANTHER. The list shows ontology terms overrepresented in the secretomes of VWAT-MSCS, sWATMSCs, and BM-MSCs taken from ND- and HFD-treated mice. Ontology terms were classified as: cellular components, protein classes, molecular functions, biological processes, and pathways.

Additional file 4. Reactome analysis. The report of pathway analysis of proteins present in the secretomes of WWAT-MSCs, sWAT-MSCs, and BMMSCs isolated from samples taken from ND- and HFD-treated mice.

\section{Abbreviations}

BM: Bone marrow; BM-MSC: Bone marrow mesenchymal stromal cells; ECM: Extracellular matrix; GO: Gene ontology; HFD: High fat diet; LCMS: Liquid chromatography-mass spectrometry; ND: Normal diet; SWAT: Subcutaneous white adipose tissue; sWAT-MSC: Subcutaneous white adipose tissue-derived mesenchymal stromal cells; vWAT: Visceral white adipose tissue; vWAT-MSC: Visceral white adipose tissue-derived mesenchymal stromal cells

\section{Acknowledgments}

Postdoc Fellowship (Assegno di Ricerca Valere 2017) from University of Campania Luigi Vanvitelli to A.N.

\section{Authors' contributions}

Conceptualization: U.G., S.O., and S.A.; Data curation: N.A., G.D.B., S.O., S.A., and D.A.; Funding acquisition: U.G. and G.P.; Investigation: M.B.A., D.A., and N.A.; Methodology: T.S., M.B.A., D.S., and N.A.; Supervision: U.G., S.O., and G.P.; Validation: N.A., M.B.A., D.S., and G.D.B.; Writing-original draft: U.G.; Writing-review \& editing: U.G., S.O., and G.P. All authors read and approved the final manuscript.

\section{Funding}

This work was partially supported by 2017-2018 Grants of the Experimental Medicine Department of University of Campania Luigi Vanvitelli to U.G.; by Regione Campania Progetto POR "Sviluppo di nanotecnologie Orientate alla Rigenerazione e Ricostruzione tissutale, Impiantologia e Sensoristica Odontoiatria/oculistica - SORRISO" CUP B23D18000250007 to G.P. and U.G..

\section{Availability of data and materials}

The datasets used and/or analysed during the current study are available from the corresponding author on reasonable request.

\section{Ethics approval}

As the study involved animals, it was approved by the Italian Ministry of Health (n. 317-2016PR), and mice were handled in compliance with the protocols approved by the Animal Care and Use Committee of University Campania Luigi Vanvitelli. 


\section{Consent for publication}

Not applicable.

\section{Competing interests}

The authors declare no competing interests.

\section{Author details}

'Department of Molecular Biology and Genetics, Faculty of Life and Natural Science, Abdullah Gül University, Kayseri, Turkey. ${ }^{2}$ Department of Experimental Medicine, Luigi Vanvitelli Campania University, Naples, Italy. ${ }^{3}$ Genome and Stem Cell Center (GENKOK), Erciyes University, Kayseri, Turkey. ${ }^{4}$ Department of Biology, Faculty of Sciences; Erciyes University, Kayseri, Turkey. ${ }^{5}$ Research Institute on Ecosystems (IRET); CNR, Naples, Italy. ${ }^{6}$ Sbarro Institute for Cancer Research and Molecular Medicine, Center for Biotechnology, Temple University, 1900 N. 12th St, Philadelphia, PA 19107-6799, USA.

Received: 22 April 2020 Accepted: 23 June 2020

\section{Published online: 29 July 2020}

\section{References}

1. Galderisi U, Giordano A. The gap between the physiological and therapeutic roles of mesenchymal stem cells. Med Res Rev. 2014;34:1100-26.

2. Park KS, Bandeira E, Shelke GV, Lasser C, Lotvall J. Enhancement of therapeutic potential of mesenchymal stem cell-derived extracellular vesicles. Stem Cell Res Ther. 2019:10:288.

3. Squillaro T, Peluso G, Galderisi U. Clinical trials with Mesenchymal stem cells: An update. Cell Transplant. 2016:25:829-48.

4. Alhattab D, Jamali F, Ali D, Hammad H, Adwan S, Rahmeh R, Samarah O, Salah B, Hamdan M, Awidi A. An insight into the whole transcriptome profile of four tissue-specific human mesenchymal stem cells. Regen Med. 2019;14:841-65.

5. Kaviani M, Azarpira N, Aghdaie MH, Esfandiari E, Geramizadeh B, Nikeghbalian S, Dehghani M. Comparison of human Mesenchymal stem cells derived from various compartments of human adipose tissue and tunica adventitia layer of the arteries subsequent to organ donation. Int J Organ Transplant Med. 2019:10:65-73.

6. Khalil C, Moussa M, Azar A, Tawk J, Habbouche J, Salameh R, Ibrahim A, Alaaeddine N. Anti-proliferative effects of mesenchymal stem cells (MSCs) derived from multiple sources on ovarian cancer cell lines: an in-vitro experimental study. J Ovarian Res. 2019;12:70.

7. Xu L, Liu Y, Sun Y, Wang B, Xiong Y, Lin W, Wei Q, Wang H, He W, Wang B, Li G. Tissue source determines the differentiation potentials of mesenchymal stem cells: a comparative study of human mesenchymal stem cells from bone marrow and adipose tissue. Stem Cell Res Ther. 2017; 8:275

8. Pawar AS, Eirin A, Tang H, Zhu XY, Lerman A, Lerman LO. Upregulated tumor necrosis factor-alpha transcriptome and proteome in adipose tissuederived mesenchymal stem cells from pigs with metabolic syndrome. Cytokine. 2020;130:155080.

9. Vizoso FJ, Eiro N, Costa L, Esparza P, Landin M, Diaz-Rodriguez P, Schneider J, Perez-Fernandez R. Mesenchymal stem cells in homeostasis and systemic diseases: hypothesis, evidences, and therapeutic opportunities. Int J Mol Sci. 2019;20.

10. Pitt JM, Kroemer G, Zitvogel L. Extracellular vesicles: masters of intercellular communication and potential clinical interventions. J Clin Invest. 2016;126: 1139-43.

11. Gowen A, Shahjin F, Chand S, Odegaard KE, Yelamanchili SV. Mesenchyma stem cell-derived extracellular vesicles: challenges in clinical applications. Front Cell Dev Biol. 2020;8:149.

12. Kusminski $C M$, Bickel $P E$, Scherer PE. Targeting adipose tissue in the treatment of obesity-associated diabetes. Nat Rev Drug Discov. 2016;15: 639-60.

13. Scherer PE. Adipose tissue: from lipid storage compartment to endocrine organ. Diabetes. 2006;55:1537-45

14. Mi H, Muruganujan A, Huang X, Ebert D, Mills C, Guo X, Thomas PD. Protocol update for large-scale genome and gene function analysis with the PANTHER classification system (v.14.0). Nat Protoc. 2019;14:703-21.

15. Jassal B, Matthews L, Viteri G, Gong C, Lorente P, Fabregat A, Sidiropoulos K, Cook J, Gillespie M, Haw R, et al. The reactome pathway knowledgebase. Nucleic Acids Res. 2020;48:D498-503.
16. Sidiropoulos K, Viteri G, Sevilla C, Jupe S, Webber M, Orlic-Milacic M, Jassal B, May B, Shamovsky V, Duenas C, et al. Reactome enhanced pathway visualization. Bioinformatics. 2017;33:3461-7.

17. Febbraio MA. Role of interleukins in obesity: implications for metabolic disease. Trends Endocrinol Metab. 2014;25:312-9.

18. Rozengurt E, Walsh JH. Gastrin, CCK, signaling, and cancer. Annu Rev Physiol. 2001;63:49-76

19. Margottin-Goguet F, Hsu JY, Loktev A, Hsieh HM, Reimann JD, Jackson PK. Prophase destruction of Emi1 by the SCF (betaTrCP/Slimb) ubiquitin ligase activates the anaphase promoting complex to allow progression beyond prometaphase. Dev Cell. 2003;4:813-26.

20. Golebiewska EM, Poole AW. Platelet secretion: from haemostasis to wound healing and beyond. Blood Rev. 2015;29:153-62.

21. Filgueiras $L R$, Serezani $\mathrm{CH}$, Jancar S. Leukotriene $B 4$ as a potential therapeutic target for the treatment of metabolic disorders. Front Immunol. 2015;6:515.

22. Cannarile MA, Weisser $M$, Jacob W, Jegg AM, Ries CH, Ruttinger D. Colonystimulating factor 1 receptor (CSF1R) inhibitors in cancer therapy. J Immunother Cancer. 2017:5:53.

23. Ali SS, Ahsan H, Zia MK, Siddiqui T, Khan FH. Understanding oxidants and antioxidants: classical team with new players. J Food Biochem. 2020:44: e13145.

24. O'Brien L, Hosick PA, John $K$, Stec DE, Hinds TD Jr. Biliverdin reductase isozymes in metabolism. Trends Endocrinol Metab. 2015;26:212-20.

25. Seiler SE, Martin OJ, Noland RC, Slentz DH, DeBalsi KL, Ilkayeva OR, An J, Newgard CB, Koves TR, Muoio DM. Obesity and lipid stress inhibit carnitine acetyltransferase activity. J Lipid Res. 2014;55:635-44.

26. Kumari B, Yadav UCS. Adipokine Visfatin's role in pathogenesis of Diabesity and related metabolic derangements. Curr Mol Med. 2018;18:116-25.

27. Marmugi A, Parnis J, Chen X, Carmichael L, Hardy J, Mannan N, Marchetti P, Piemonti L, Bosco D, Johnson $P$, et al. Sorcin links pancreatic beta-cell lipotoxicity to ER Ca2+ stores. Diabetes. 2016;65:1009-21.

28. De Falco S. The discovery of placenta growth factor and its biological activity. Exp Mol Med. 2012:44:1-9.

29. La Paglia L, Listi A, Caruso S, Amodeo V, Passiglia F, Bazan V, Fanale D. Potential role of ANGPTL4 in the cross talk between metabolism and Cancer through PPAR signaling pathway. PPAR Res. 2017;2017:8187235.

30. Sheng J, Xu Z. Three decades of research on angiogenin: a review and perspective. Acta Biochim Biophys Sin Shanghai. 2016;48:399-410.

31. Brandt $\mathrm{C}$, Hansen $\mathrm{RH}$, Hansen JB, Olsen $\mathrm{CH}$, Galle $\mathrm{P}$, Mandrup-Poulsen $\mathrm{T}$ Gehl J, Pedersen BK, Hojman P. Over-expression of Follistatin-like 3 attenuates fat accumulation and improves insulin sensitivity in mice. Metabolism. 2015;64:283-95.

32. Maguer-Satta $V$, Rimokh R. FLRG, member of the follistatin family, a new player in hematopoiesis. Mol Cell Endocrinol. 2004;225:109-18.

33. Nam J, Perera P, Gordon $R$, Jeong YH, Blazek AD, Kim DG, Tee BC, Sun Z, Eubank TD, Zhao $Y$, et al. Follistatin-like 3 is a mediator of exercise-driven bone formation and strengthening. Bone. 2015;78:62-70

34. Franklin CC, Backos DS, Mohar I, White CC, Forman HJ, Kavanagh TJ. Structure, function, and post-translational regulation of the catalytic and modifier subunits of glutamate cysteine ligase. Mol Asp Med. 2009;30:86-98.

35. Zhou Y, Kok KH, Chun AC, Wong CM, Wu HW, Lin MC, Fung PC, Kung H, Jin DY. Mouse peroxiredoxin $\mathrm{V}$ is a thioredoxin peroxidase that inhibits p53induced apoptosis. Biochem Biophys Res Commun. 2000;268:921-7.

36. Alessio N, Squillaro T, Di Bernardo G, Galano G, De Rosa R, Melone MA Peluso G, Galderisi U. Increase of circulating IGFBP-4 following genotoxic stress and its implication for senescence. Elife. 2020;9.

37. Ludvigsen TP, Olsen LH, Pedersen HD, Christoffersen BO, Jensen LJ. Hyperglycemia-induced transcriptional regulation of ROCK1 and TGM2 expression is involved in small artery remodeling in obese diabetic Gottingen Minipigs. Clin Sci (Lond). 2019;133:2499-516.

38. Vermeren M, Lyraki R, Wani S, Airik R, Albagha O, Mort R, Hildebrandt F, Hurd T. Osteoclast stimulation factor 1 (Ostf1) KNOCKOUT increases trabecular bone mass in mice. Mamm Genome. 2017:28:498-514.

39. Fan J, Fong T, Chen X, Chen C, Luo P, Xie H. Glia maturation factor-beta: a potential therapeutic target in neurodegeneration and neuroinflammation. Neuropsychiatr Dis Treat. 2018;14:495-504.

40. Danilova T, Galli E, Pakarinen E, Palm E, Lindholm P, Saarma M, Lindahl M. Mesencephalic astrocyte-derived Neurotrophic factor (MANF) is highly expressed in mouse tissues with metabolic function. Front Endocrinol (Lausanne). 2019;10:765 
41. Liao R, Ren G, Liu H, Chen X, Cao Q, Wu X, Li J, Dong C. ME1 promotes basal-like breast cancer progression and associates with poor prognosis. Sci Rep. 2018;8:16743.

42. Yang K, Adin C, Shen Q, Lee LJ, Yu L, Fadda P, Samogyi A, Ham K, Xu L, Gilor C, Ziouzenkova $\mathrm{O}$. Aldehyde dehydrogenase 1 a1 regulates energy metabolism in adipocytes from different species. Xenotransplantation. 2017;24.

43. Jones DL, Wagers AJ. No place like home: anatomy and function of the stem cell niche. Nat Rev Mol Cell Biol. 2008;9:11-21.

44. Kumar A, Kumar V, Rattan V, Jha V, Pal A, Bhattacharyya S. Molecular spectrum of secretome regulates the relative hepatogenic potential of mesenchymal stem cells from bone marrow and dental tissue. Sci Rep. 2017;7:15015.

45. Nakanishi C, Nagaya N, Ohnishi S, Yamahara K, Takabatake S, Konno T, Hayashi K, Kawashiri MA, Tsubokawa T, Yamagishi M. Gene and protein expression analysis of mesenchymal stem cells derived from rat adipose tissue and bone marrow. Circ J. 2011;75:2260-8.

46. Niada S, Giannasi C, Ferreira LM, Milani A, Arrigoni E, Brini AT. 17betaestradiol differently affects osteogenic differentiation of mesenchymal stem/ stromal cells from adipose tissue and bone marrow. Differentiation. 2016;92: 291-7.

47. Jeon YJ, Kim J, Cho JH, Chung HM, Chae Jl. Comparative analysis of human Mesenchymal stem cells derived from bone marrow, placenta, and adipose tissue as sources of cell therapy. J Cell Biochem. 2016;117:1112-25.

48. Nahar S, Nakashima Y, Miyagi-Shiohira C, Kinjo T, Kobayashi N, Saitoh Watanabe M, Noguchi H, Fujita J. A comparison of proteins expressed between human and mouse adipose-derived Mesenchymal stem cells by a proteome analysis through liquid chromatography with tandem mass spectrometry. Int J Mol Sci. 2018;19.

49. Hardouin P, Pansini V, Cortet B. Bone marrow fat. Joint Bone Spine. 2014;81: 313-9.

50. Blobel G, Dobberstein B. Transfer of proteins across membranes. I. Presence of proteolytically processed and unprocessed nascent immunoglobulin light chains on membrane-bound ribosomes of murine myeloma. J Cell Biol. 1975;67:835-51.

51. Schmidt RL, Simonovic M. Synthesis and decoding of selenocysteine and human health. Croat Med J. 2012;53:535-50.

52. Jackson E, Shoemaker R, Larian N, Cassis L. Adipose tissue as a site of toxin accumulation. Compr Physiol. 2017;7:1085-135.

53. Rosen ED, Spiegelman BM. Molecular regulation of adipogenesis. Annu Rev Cell Dev Biol. 2000;16:145-71.

54. Lattanzi W, Geloso MC. Editorial: crosstalk between the Osteogenic and neurogenic stem cell niches: how far are they from each other? Front Cell Neurosci. 2015;9:504.

55. Utsuyama M, Shiraishi J, Takahashi H, Kasai M, Hirokawa K. Glia maturation factor produced by thymic epithelial cells plays a role in T cell differentiation in the thymic microenvironment. Int Immunol. 2003:15:55764

\section{Publisher's Note}

Springer Nature remains neutral with regard to jurisdictional claims in published maps and institutional affiliations.

Ready to submit your research? Choose BMC and benefit from:

- fast, convenient online submission

- thorough peer review by experienced researchers in your field

- rapid publication on acceptance

- support for research data, including large and complex data types

- gold Open Access which fosters wider collaboration and increased citations

- maximum visibility for your research: over $100 \mathrm{M}$ website views per year

At $\mathrm{BMC}$, research is always in progress.

Learn more biomedcentral.com/submissions 